\title{
Le miel et le fiel : des excès de bouche dans Timon of Athens
}

\section{Nathalie Vienne-Guerrin}

\section{(2) OpenEdition}

\section{Journals}

\section{Édition électronique}

URL : http://journals.openedition.org/shakespeare/1038

DOI : 10.4000/shakespeare.1038

ISSN : 2271-6424

\section{Éditeur}

Société Française Shakespeare

\section{Édition imprimée}

Date de publication : 1 novembre 2007

Pagination : 206-222

ISBN : 2-9521475-4-X

\section{Référence électronique}

Nathalie Vienne-Guerrin, «Le miel et le fiel : des excès de bouche dans Timon of Athens », Actes des congrès de la Société française Shakespeare [En ligne], 25 | 2007, mis en ligne le 10 février 2008, consulté le 20 avril 2019. URL : http://journals.openedition.org/shakespeare/1038 ; DOI : 10.4000/ shakespeare. 1038 


\section{Shakespeare et l'excès}

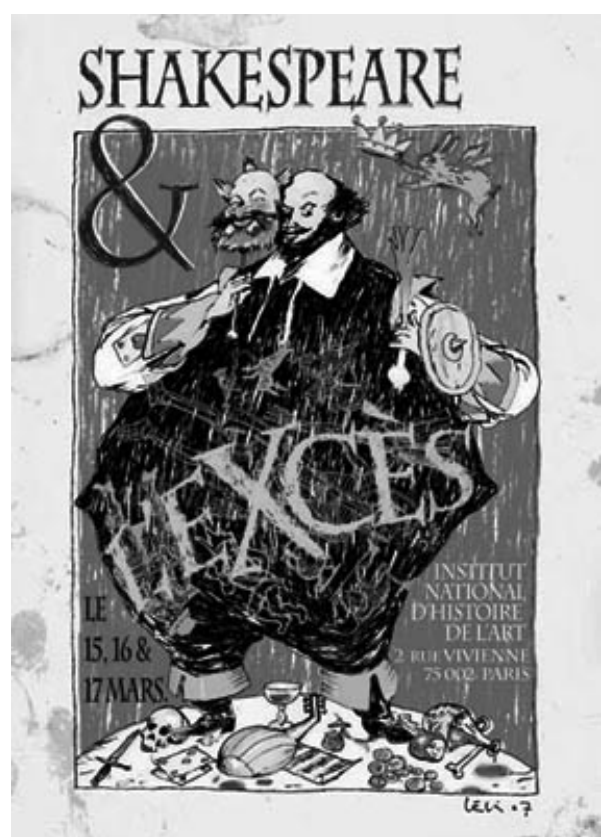

actes du Congrès

organisé par la

SOCIÉTÉ FRANÇAISE SHAKESPEARE

les 15,16 et 17 mars 2007

textes réunis par

Pierre KAPITANIAK

sous la direction de

Jean-Michel DÉPRATS 
COUVERTURE :

Edouard Lekston, Falstaff, 2007

conception graphique et logo

Pierre Kapitaniak

\section{(C) 2007 Société Française Shakespeare \\ Institut du Monde Anglophone}

Université de Paris III - Sorbonne Nouvelle

5 rue de l'École de Médecine 75006 Paris

www.societefrancaiseshakespeare.org

Tous droits de traduction, de reproduction et d'adaptation réservés pour tous les pays 


\title{
LE MIEL ET LE FIEL : DES EXCÈS DE BOUCHE DANS TIMON OF ATHENS
}

\author{
Nathalie VIENNE-GUERRIN
}

Nous analyserons les deux principaux excès de langue qui se manifestent abondamment dans Timon of Athens, la flatterie et l'imprécation. De "mauvaise oreille " ouverte à la flatterie, Timon devient "mauvaise langue " qui se laisse aller aux (dé)plaisirs de l'exécration et de la vitupération. Nous présenterons les grands traits qui se dégagent de la représentation de la flatterie à l'époque élisabéthaine en mobilisant des sources philosophiques, morales et iconographiques et montrerons combien la pièce est imprégnée de cette "culture de la flatterie ". Nous analyserons le fonctionnement rhétorique et dramatique des débordements verbaux de Timon en les contrastant notamment avec la langue du cynique Apemantus. Nous suggèrerons que paradoxalement, mais conformément aux représentations de l'époque, le miel de la lanque s'avère, dans Timon of Athens, plus destructeur, plus malfaisant, plus efficace que l'évacuation du fiel par voie d'injure, d'imprécation ou de malédiction. Nous analyserons également les rapports que ces excès de langue entretiennent avec le corps et notamment avec l'alimentaire, dans une pièce où la langue se présente comme un organe véritablement carnassier.

$\mathrm{M}$

éfie-toi, Timon, tu es en train de te faire bouffer ! »: voilà en quels termes nous pourrions résumer les mises en garde qu'Apemantus sert et ressert à Timon au cours du banquet initial. La première partie de la pièce suggère à maintes reprises l'image d'un Timon dévoré par les flatteurs qui l'entourent, tel Actéon par ses chiens ${ }^{1}$ : «What a number of men eats Timon, and he sees 'em not ! » (I.ii.39) ${ }^{2}$. Dans Tim on of Athens, la flatterie, considérée à l'époque de Shakespeare comme l'un des pires péchés de la langue car elle a la perfidie d'aller masquée, la flatterie est présentée comme un excès de table. Les visiteurs qui défilent chez Timon («this great flood of visitors», I.i.43) sont des goinfres, (« riotous $^{3}$ feeders»,

\footnotetext{
${ }^{1}$ La figure d'Actéon est régulièrement associée à la flatterie dans les textes élisabéthains. À ce sujet, voir Nathalie Vienne-Guerrin, "Coriolanus or the "Arraignment of an Unruly Tongue" », in Coriolan de William Shakespeare. Langages, Interprétations, Politique(s), éd. Richard Hillman, Tours, 2007, p. 139-142.

2 Pour les citations, nous utilisons l'édition Cambridge, Timon of Athens, éd. Karl Klein, Cambridge, Cambridge University Press, 2001, mais nous mobiliserons aussi ponctuellement l'édition Oxford de John Jowett, William Shakespeare and Thomas Middleton, The Life of Tim on of Athens, Oxford University Press, 2004.

3 Dans Timon of Athens, l'excès est évoqué à plusieurs reprises par le terme «riot» qui signifie «Wanton, loose, or wasteful living; debauchery, dissipation, extravagance » (OED, 1.a.) ou encore "An instance or course of loose living; a noisy feast or wanton revel; a disturbance arising from this ; an extravagant display of something » $(O E D, 2$.a.). Flavius fait allusion aux dépenses de Timon en utilisant l'expression «flow of riot » (II. ii. 3). Timon
} 
II.ii.153), des bouches cannibales ${ }^{4}$ et gloutonnes (« glutt'nous maws », III.iv.50), des sangsues qui, à force de «lèche ${ }^{5}$ », absorbent, engloutissent (« englut», II.ii.160) la substance de leur hôte.

Mais Apemantus nous rappelle également que le flatté ne vaut pas mieux que le flatteur: "He that loves to be flattered is worthy o'th'flatterer» (I.i.227). L'oreille avide qui absorbe les mots doux est tout aussi coupable que la bouche qui les lui offre. L'oreille du flatté est aussi gourmande que la bouche du flatteur. Aussi peut-on entendre dans les propos d'Apemantus une mise en garde inverse : "Méfie-toi, Timon, tu manges trop de bonbons». Le «What a number of men eats Timon », avec son verbe au singulier (« eats ») pourrait bien inverser, ou en tout cas brouiller, le rapport entre le mangeur et le mangé6. Ce dont Timon se repaît, dans la première partie de la pièce, c'est du miel de la flatterie 7 . Timon d'Athènes, pourrait-on dire, aime trop les douceurs. Notre propos est de suggérer que ce que Timon ingère dans la première partie de la pièce, il le vomit, il le recrache, dans la deuxième.

Dès le début de la pièce, c'est en termes d'excès que Timon nous est décrit: «He passes» (I.i.12), "He outgoes the very heart of kindness » (I.i.274), « He pours it out » (I.i.275) ${ }^{8}$. Lorsque Apemantus le rejoint dans le «no man's land» où il s'est réfugié, il dresse le portrait d'un homme hors-norme: "The middle of humanity thou never knewest, but the extremity of both ends » (IV.iii.307-8). Timon, selon Apemantus, ne connaît pas la mesure, il ignore les limites, il explore les extrêmes. «He is a man of excess », écrit de lui Robert C.

utilise le terme à deux reprises dans un sens sexuel («and drown themselves in riot!», IV.i.28, « in general riot », IV. iii.263).

${ }^{4}$ Voir l'article de Ruth Morse, «Unfit for Human Consumption, Shakespeare's Unnatural Food », Deutsche Shakespeare Gesellshaft West Jahrbuch, Bochum, 1983, p. 125-149.

${ }^{5}$ L'image finale de la pièce, «each other's leech» (v.iv.84), est emblématique de l'importance de ce motif.

${ }^{6}$ Sur la consommation réciproque, voir l'article de Daniel W. Ross, «What a number of men eats Timon : Consumption in Timon of Athens », Iow a State Journal of Research, vol. $59, \mathrm{n}^{\circ} 3$, février 1985 , p. $273-284$

${ }^{7}$ Pour une analyse de l'imaginaire shakespearien de la flatterie, voir l'article de C. H. Hobday, "Why the sweets melted: a study in Shakespeare's Imagery», Shakespeare Quarterly, vol. 16, ${ }^{\circ} 1$, hiver 1965, p. 3-17.

${ }^{8}$ Cette question de l'excès est abordée sous un autre angle dans l'article de Richard Marienstras, "Amitié et démesure dans Timon d'Athènes", in Shakespeare, La Renaissance et l'amitié, éd. Richard Marienstras et Dominique Goy-Blanquet, Presses de l'UFR de Langues Université de Picardie, 1998, p. 271-286. 
Elliott dans The Power of Satire $(1960)^{9}$, décrivant ainsi un personnage dont les excès le placent à la limite du sublime et du grotesque, du grandiose ${ }^{10}$ et du ridicule. Richard Fly parle quant à lui de « The unmediated world of Timon of Athens $»(1976)^{11}$, tandis que Frank Kermode écrit : " The play was evidently designed to consist of two halves illustrating contrasting modes of excess ${ }^{12} »$.

Nous voudrions suggérer que la racine commune à ces excès se trouve dans la langue, et que ce que la pièce met en scène, c'est un dérèglement, voire une déréglementation de la bouche. Dans Timon of Athens, on va d'un excès de bouche à l'autre, de l'ingestion à l'expulsion. Ce qui est souvent présenté comme un parcours qui mène de la philanthropie à la misanthropie, d'un trop plein de générosité («bounty») à un débordement de haine, ce parcours me semble pouvoir être lu comme la chronique d'une indigestion, l'histoire fantastique de la métamorphose du miel en fiel. Sans doute autant que de colère ou de haine, Timon est pris de nausées : «I am sick of this false world» (IV.iii.371). Les débordements verbaux, les invectives, malédictions, imprécations, injures déversées dans la deuxième partie de la pièce ont des allures de déchets rejetés par un organisme qui «exècre» et «excrète» la société dont il se régalait jadis. Avant le dégoût, il y a eu le goût. Si Timon of Athens peut offrir une réflexion sur «l'homme et l'excretum ${ }^{13} »$, pour reprendre le titre d'un texte bien connu de Claude Gaignebet ${ }^{14}$, cette pièce met aussi en scène un personnage gargouille. Timon, c'est cette gorge, évoquée par Éric Beaumartin et Michel Garcia dans un ouvrage consacré à l'Invective au Moyen Âge, c'est cette gueule qui «chasse vers le dehors [...] une matière que le dedans refuse ${ }^{15} »$.

\footnotetext{
${ }^{9}$ Robert C. Elliott, The Power of Satire: Magic, Ritual, Art, Princeton, Princeton University Press, 1960, p. 156.

10 Ibid., p. 159.

${ }^{11}$ Shakespeare's Mediated World, Amherst, University of Massachusetts Press, 1976, p. $119-142$.

12 The Riverside Shakespeare, 2 éd., Boston, New York, Houghton Mifflin Company, 1997, p. 1491.

13 Comme l'a suggéré notamment Maurice Hunt dans «The Backward Voice of Coriolanus », Shakespeare Studies 31 (2004), p. 220-239.

14 Claude Gaignebet et Marie-Claude Perier, «L'homme et l'excretum », in Histoire des Ma urs, vol. 1, La Pléiade, 1990, p. 831-893.

${ }^{15}$ Dans L'Invective au Moyen Âge, France, Espagne, Italie (Atalaya, Revue Française d'Études Médiévales Hispaniques, n5, 1994, Paris, Presses de la Sorbonne Nouvelle, 1995), Beaumartin et Garcia notent l'importance de la figure de la gargouille pour évoquer les
} 
Après avoir montré comment, dans le monde de Shakespeare, l'imaginaire de la bouche et de la langue s'inscrit dans une problématique de l'excès et du contrôle et soude les deux fonctions de la langue que sont le parler et le manger, nous verrons comment le texte dessine le motif d'une bouche qui après s'être régalée du sucre de la flatterie, dé-gueule sur le monde des flots d'invectives et de malédictions. Considérée à la lumière de cette pièce, l'expression «vomir des imprécations» prend un sens plus que métaphorique. Enfin nous analyserons comment, à la fin de la pièce, l'écrit prend le relais des cris, et comment les épitaphes figent et propagent tout à la fois les excès de langue qui ont rempli la pièce.

\section{La bouche et ses gardes $^{16}$}

La nature (ou devrait-on dire Dieu), dans sa grande bonté, nous a donné une langue et elle a pris la peine, semble-t-il, d'inscrire dans ce qu'Érasme appelait ce «tout petit organe tout flasque ${ }^{17}$ » les moyens de son propre contrôle ${ }^{18}$. La langue est petite, ce qui signifie que les propos qu'elle émet doivent être mesurés. La langue est une, ce qui indique qu'il est interdit d'en faire double usage, et qu'elle ne doit pas souffler, comme le voyageur dans la fable du satyre d'Ésope ${ }^{19}$, à la fois

excès ou péchés de la langue (p. 262). Leurs analyses sont prolongées et citées dans l'ouvrage coordonné par Didier Girard et Jonathan Pollock, L'Invective, Quand le corps reprend la parole, Presses Universitaires de Perpignan, 2006, p. 19.

${ }^{16}$ L'expression « garde de la langue » est utilisée par Carla Casagrande et Silvana Vecchio, Les Péchés de la Langue. Discipline et éthique de la parole dans la culture médiévale, Les Éditions du Cerf, 1991, p. 42 et passim.

${ }^{17}$ Érasme, La Langue, éd. et trad. Jean-Paul Gillet, Genève, Labor et Fides, 2002, p. 170.

18 Dans un chapitre intitulé "Sins of the tongue", dans The Body in Parts, Fantasies of Corporeality in Early Modern Europe, éd. David Hillman et Carla Mazzio, Routledge, 1997, p. 67, Carla Mazzio exprime cette idée en ces termes : «Nature, according to Erasmus, Brathwait, and many other early modern anatomists of the word, has encoded mechanisms for censorship in to the anatomical structure of man. »

${ }^{19}$ Voir Aesop's Fables, trad. Laura Gibbs, Oxford World's Classics, 2002, p. 173-174, "Fable of the Satyr and his Guest». Cette fable est citée notamment par Jean de Marconville dans son traité (traduit du français) A Treatise of the Good and Evell Toungue, ca. 1592 (STC 17313), C5. Marconville emprunte cette histoire à Érasme, La Langue, p. 243. On trouve la même histoire dans les Adages d'Erasme, Adage 1.viii. 30 (LB II 309B) intitulé : «Ex eodem ore calicum et frigidum efflare (Out of one mouth to blow hot and cold) », Collected Works of Erasmus 32, p. 139-140. Voir Avianus, Fables, in Minor Latin Poets, vol. 2, trans. J. Wight Duff and Arnold M. Duff, p. 669-749, Loeb Classical Library, Harvard University Press, 1934, Fable 29, « The traveller and the Satyr », p. 727. Cette fable est reprise par Geffrey Whitney, A Choice of Emblemes [1586], intr. John Manning, Aldershot, Scolar Press, 1989, p. 160 («Bilingues Cavendi »). 
le chaud et le froid. La nature nous a également fourni la double barrière des dents et des lèvres, deux «garde bouches » qui devraient permettre à la langue de ne pas s'égarer ${ }^{20}$. Voilà, à grands traits, comment les textes élisabéthains consacrés à la langue décryptent l'anatomie de cet organe.

Mais ces mêmes textes suggèrent que malgré les barrières que la nature a pris soin d'installer, la langue semble ne pouvoir jamais être contenue. «And the tongue can no man tame»: cette citation biblique, tirée de l'épître de saint Jacques (3.8) analysée notamment par Thomas Adams dans son sermon «The Taming of the Tongue» $(1614)^{21}$, est recyclée et commentée à l'infini dans les traités de l'époque qui dénoncent les excès de la langue (notamment la flatterie, l'injure, la calomnie, le blasphème, la moquerie, la médisance, le mensonge, le bavardage), et qui tentent d'offrir à l'homme les remèdes à ces excès. En inscrivant dans la physiologie de la langue les instruments de son contrôle, la nature semble l'avoir du même coup, hélas, programmée pour dépasser les bornes. Qui dit limite dit transgression.

D'autre part, si la langue a besoin de barrières, c'est parce qu'elle a, par nature, tendance à déraper. Dans son anthologie de pensées intitulée A Treatise of Morall Philosophie, best-seller élisabéthain, William Baldwin ouvre son chapitre consacré à la langue en ces termes:

The tongue is a slippery and nimble instrument, wherby commonly the treasures of the heart are in such wise unlocked, layed forth and spread abroad, that not onely thereby friendship is greatly ingendred, earthly

\footnotetext{
20 Cette image des lèvres et des dents comme doubles barrières est un leitmotiv qui apparaît dans la plupart des traités de la langue de l'époque. Voir notamment Marconville, A Treatise: "nature hath enclosed and hedged in with teeth and lips, as it were within a defensible bulwarke, that it might not be ranging unadvisedly ». Marconville emprunte l’idée à Érasme, La Langue, p. 86-87: «La nature a placé sur son chemin le double rempart et la barrière des trente-deux dents. Homère par exemple utilise une expression pleine de sens quand il parle de «l'enclos des dents» [Od., I. 64, X. 328] [...] La nature ensuite a aussi placé en avant les doubles battants des lèvres, signalant à l'évidence que dans la langue, qu'elle a ainsi cachée, se trouve un précieux trésor, mais qu'il y a un très grand danger à en user de manière intempestive et immodérée. » Voir aussi William Perkins, A Direction for the Government of the Tongue, Londres, 1595 [1593], STC 19760.5, p. 6 : «The tongue is placed in the middle of the mouth, and it is compassed in with lippes [...] and teeth as with a double trench, to shew us, how we are to use heed and preconsideration before wee speake » et Thomas Adams, "The Taming of the Tongue», The Works of Thomas Adams, vol. 3, Eureka, Tanski Publications, 1998, p. 10-22: «A double fence hath the Creator given to confine it, the lips and the teeth; that through these mounds it might not break» (p. 14).

${ }^{21}$ The Works of Thomas Adams, p. 10-22.
} 
Treasures increased, the life quietly stablished, perpetuall praise and everlasting felicitie obtayned, but contrariwise frienship is decayed, worldly riches are diminished, the life most miserably wasted, infamy and immortall payne is thereby purchased ${ }^{22}$.

Ce passage résume deux des traits saillants de la représentation de la langue dans la culture élisabéthaine. La langue est un terrain glissant («slippery»), aussi «traître» que les flatteurs qui sont décrits dans Timon of Athens comme des «glib and slippery creatures»(I.i.55). Cette tendance au dérapage incontrôlé est elle aussi inscrite dans la physiologie de la langue, comme le soulignent Carla Casagrande et Silvana Vecchio: «la mobilité de la langue, située dans un endroit humide et glissant, représente une possibilité permanente de péché ${ }^{23}$ », autant dire d'excès. Il n'est pas fortuit que l'une des figures récurrentes mobilisées dans les traités de la langue soit celle d'Anacharsis. Représenté une main sur la bouche, l'autre sur une partie plus basse de son anatomie ${ }^{24}$, cette figure indique clairement quels sont les deux organes les plus propices à l'excès. L'exemple de Pythagore qui imposait à ses disciples des années de silence (de deux à cinq ans, selon les sources) était également constamment utilisé pour indiquer la nécessité du contrôle de la langue et les vertus du silence. Il est un autre élément anatomique qui explique que la bouche ait tendance aux excès : c'est qu'en elle circulent à la fois les mets et les mots $^{25}$. Dans sa grande parcimonie, la nature a en effet donné à la langue la double fonction du parler et du manger ${ }^{26}$. Un seul organe pour deux fonctions : voilà qui devrait signaler à l'homme qu'il doit

22 Baldwin, William. A Treatise of Morall Philosophie (1547) by William Baldwin. Enlarged by Thomas Palfreyman, a facsimile reproduction of the edition of 1620 with an introduction by Robert Hood Bowers. Gainesville, Florida, Scholars' Facsimiles \& reprints, 1967, p. 320 (Chap. «Of the Tongue, Detraction, Speech and Silence »)

23 Casagrande et Vecchio, op. cit., p. 98.

24 Voir Guillaume de la Perrière, Morosophie, Lyon, Macé Bonhomme, 1553, Quatrain 16 : «Anacharsis de tenir apprenoit / La main senestre à la partie honteuse, / Et la main dextre à la bouche tenoit, / Comme plus forte, en la part plus douteuse. »

25 Nous empruntons l'expression à Michel Jeanneret, Des Mets et des Mots. Banquets et Propos de Table à la Renaissance, Paris, Librairie José Corti, 1987.

${ }^{26}$ Dans l'Angleterre de Shakespeare mais aussi au Moyen Âge, c'est cette vision aristotélicienne de la double fonction de la langue qui domine. Voir Aristote, De L'Âme, II, 8, 420b: «En effet, la nature se sert de l'air respiré en vue de deux fins, comme elle se sert de la langue à la fois en vue du goût et en vue du langage articulé : de ces deux dernières fonctions, le goût est nécessaire à la vie (c'est pourquoi d'ailleurs il appartient à un plus grand nombre d'animaux), alors que l'expression de la pensée n'est qu'en vue du bienêtre...» (trad. Jean Tricot, Paris, Librairie Philosophique J. Vrin, 1995 (1934), p. 120. À ce sujet, voir Carla Casagrande et Silvana Vecchio, op. cit., p. 117-122. 
gérer avec mesure à la fois ce qui entre et ce qui sort de sa bouche. Mais, dans sa grande imprudence, la nature a par là même fait de la bouche une dangereuse zone de «transit ${ }^{27}$ », une porte d'entrée et de sortie, un carrefour où se croisent l'alimentaire et le verbal et où les risques d'accidents sont grands.

Ressort également de l'extrait de Baldwin précédemment cité l'idée que, comme Timon, la langue ne connaît pas de juste mesure mais seulement les extrêmes («the extremity of both ends»). La culture élisabéthaine est hantée par la figure d'une langue qui est sans cesse présentée comme le meilleur et le pire organe ${ }^{28}$, source de tous les bienfaits et de tous les méfaits. Cette langue dont la Bible nous dit qu'elle a le pouvoir de vie et de mort (Prov. 18.21), cette langue semble être le lieu de tous les excès.

Deux dérèglements de la bouche me semblent être au cœur de Timon of Athens : la flatterie et la malédiction ${ }^{29}$, deux excès de gueule qui convergent dans l'image du chien qui hante toute la pièce ${ }^{30}$.

\footnotetext{
${ }^{27}$ Voir Jeanneret, p. 33. Notons qu'il semble que Timon parle le plus souvent en mangeant tout au long de la pièce.

${ }^{28}$ Voir notamment l'histoire d'Amasis et de Pittacos qui ouvre le traité d'Érasme: « Amasis, le roi d'Égypte, fit envoyer à Pittacos, l'un des sept sages célèbres de la Grèce, qui était son hôte, un animal qu'on avait immolé, lui demandant de lui retourner la partie qu'il jugerait la meilleure et aussi celle qu'il estimerait la pire ; il lui retournerait, croyait-il, deux parties différentes. L'autre coupa la langue et l'envoya au roi, ce qui signifiait qu'en l'homme il n'y avait rien de mieux qu'une bonne langue, mais rien de plus nuisible qu'une mauvaise ; car chez tous les autres êtres vivants, la langue n'est la partie ni la pire ni la meilleure.» (La Langue, p. 76-77). Cette histoire, issue d'Ésope et que l'on trouve également chez Plutarque est reprise de multiples fois dans les ouvrages de l'époque. Voir, par exemple, Jean de Marconville, op. cit., fol. A6v; Gabriel Harvey, The Trimming of Thomas Nashe, Londres, 1597, STC 12906, fol. C r ; Robert Albott, Wits theater of the little world, Londres, 1599, STC 381, p. 85 ; Giacomo Affinati, The dumbe divine speaker, or: Dumbe speaker of Divinity A learned and excellent treatise, in praise of silence: shewing both the dignitie, and defectes of the tongue. Written in Italian, by Fra. Giacomo Affinati d'Acuto Romano. And truelie translated by A.M., Londres, 1605, STC 190, p. 40 ; Joseph Swetnam, The araignment of lewd, idle, froward, and unconstant women, Londres, 1615, STC 23534, p. 41-42.

${ }^{29}$ La malédiction dans Timon a été abordée sous d'autres angles par Keir Elam, dans son lumineux article "I'll plague thee for that word": Language, Performance, and Communicable Disease », Shakespeare Survey 50, 1997, p. 19-27. Voir également William O. Scott, «The Paradox of Timon's Self-Cursing », Shakespeare Quarterly 35, 1984, p. 290 304 .

30 Voir l'étude de William Empson, The Structure of Complex Words, Londres, Chatto and Windus, 1977, chap. 8 (« Timon's dog», p. 175-184).
} 


\section{Les dérèglements de la langue: flatter et maudire}

La flatterie qui est au centre de la pièce tient une grande place dans les catalogues des excès («abuses») de langue qui sont dressés par les Élisabéthains. Et c'est en termes alimentaires et gustatifs que ce péché de la langue est le plus souvent représenté dans les textes de l'époque. La flatterie est un vice du «palais» dans les deux sens du terme. Les banquets de cour qui pourraient être des lieux de fraternité sont en effet très souvent propices aux actes de flatterie.

D'autre part, ce qui est au cœur de la représentation de la flatterie, c'est le double rapport alimentaire qui s'établit entre le flatteur et le flatté : le flatteur mange celui qu'il flatte, il se nourrit de lui, avant d'abandonner une carcasse vidée de sa substance. Dans l'iconographie de l'époque, les flatteurs sont représentés comme des parasites, des rats ou des corbeaux qui délaissent leur proie une fois que celle-ci est évidée ${ }^{31}$. Mais le flatteur nourrit également le flatté d'un sucre qui ne révèle que trop tard son arrière-goût amer. Si, dans l'imaginaire élisabéthain, le flatteur est présenté comme un caméléon qui change de couleur en fonction des situations et des interlocuteurs, comme un miroir qui s'évertue à refléter celui à qui il parle - dans Timon est évoquée la figure du « glass-faced flatterer » (I.i.60) -, il est également celui qui sert à l'autre un miel empoisonné ${ }^{32}$.

Cette double relation alimentaire entre le mangeur et le mangé est présente dans Timon of Athens où le flatteur dévore, tandis que le flatté déguste. L'oreille de Timon est une bouche qui se repaît des douces musiques de la flatterie avant de les trouver indigestes ${ }^{33}$.

Le début de la pièce montre que Timon souffre d'un dérèglement du goût. Il ne sait pas distinguer l'ami du flatteur ${ }^{34}$, il ne perçoit pas le poison qui se cache derrière le sucre qu'on lui sert. Comme le rappelle Whitney dans l'un de ses emblèmes intitulé «fel in

\footnotetext{
${ }^{31}$ Voir Vienne-Guerrin, op. cit., p. 140

32 Pour toutes ces images, voir l'article de Clifford Davidson, «Timon of Athens: The Iconography of False Friendship », The Huntington Library Quarterly, Été 1980, vol. 43, $\mathrm{n}^{\circ} 3$, p. 181-200. Voir aussi Vecchio et Casagrande, notamment le chapitre consacré à l'« adulatio », p. 253-263.

33 Ce télescopage de la bouche et de l'oreille apparaît notamment lorsque Timon propose à ses invités de les nourrir d'une musique discordante: "feast your ears with the music awhile, if they will fare so harshly o'th'trumpet's sound » (III.vi.26-28).

34 Voir le texte de Plutarque, «How to tell a flatterer from a friend» (« Quomodo adulator ab amico internoscatur »), in Moralia 1, trans. Frank Cole Babbit, Loeb Classical Library, 1927 (1949), p. 263-395 (48E-74E).
} 
melle» («poison in honey »), il y a du fiel derrière ce miel ${ }^{35}$. Et Timon of Athens retrace précisément l'histoire de cette découverte, l'histoire de ce choc gustatif. «But myself / Who had the world as my confectionary » (IV.iii.267) : le monde était sa confiserie; il était «tout miel ». Mais à manger trop de sucre, on attrape mal au cœur. L'excès de sucrerie «nuit gravement à la santé ». Apemantus l'avait pourtant prévenu :

We spend our flatteries to drink those men,

Upon whose age we void it up again

With poisonous spite and envy.

(I.ii.124-26)

Dès le début de la pièce le cynique nous offrait l'image prémonitoire de la métamorphose du miel en fiel («spite and envy»). Après avoir bu à la source de la flatterie, il faut régurgiter. Les malédictions que Timon déverse (on pourrait dire « he pours it out») sur le monde participent de cette régurgitation. «Would thou wert clean enough to spit upon» (IV.iii.355), s'exclame Timon. «A plague on thee, thou art too bad to curse» (IV.iii.356) lui répond Apemantus ${ }^{36}$. $\mathrm{Au}$ détour de cette scène de «flyting ${ }^{37}$ » où Elliott voit un basculement du sublime dans le grotesque, se trouve formulée l'équivalence du crachat («spit upon») et de la malédiction («curse»), équivalence que le crachat de Lady Anne sur Richard dans Richard III exprimait déjà ${ }^{38}$. L'image d'un Timon en proie aux excès de bouche est évoquée un peu plus tôt :

APEMANTUS. [...] Will the cold brook,

Candied with ice, caudle thy morning taste

To cure thy o'ernight's surfeit?

(IV.iii.227-29)

Lorsque Apemantus demande ironiquement à Timon si le frais ruisseau saura guérir sa gueule de bois («o'ernight's surfeit»), il fait émerger l'image d'un homme qui s'est laissé aller au trop boire et au

\footnotetext{
35 Whitney, p. 147. Pour la flatterie, voir aussi Whitney, p. 24 («Latet anguis in herba»).

${ }^{36}$ Ces répliques sont prononcées à la fin de l'échange entre Apemantus et Timon à l'acte IV, scène iii : "The scene is past rage and into the ridiculous » écrit Elliott (p. 159). Il semble qu'en passant de la malédiction à l'injure, on passe du sublime au grotesque. «Tu es trop sale pour que je te crache dessus », «Tu est trop mauvais pour que je te maudisse » : les éditeurs ont souvent résolu la contradiction d'un Apemantus qui dit qu'il ne peut maudire au moment même où il maudit en soulignant le caractère conventionnel et donc insuffisant et mort de «A plague upon thee».

${ }^{37}$ Le terme renvoie à un échange d'injure ritualisé.

38 King Richard III, I.ii.149 (éd. J anis Lull, Cambridge University Press, 1999).
} 
trop manger et qui maintenant tente de soulager sa panse pleine en vomissant tripes et boyaux sur un monde devenu indigeste. Cette sensation de dégoût apparaissait un peu plus tôt lorsque Timon évoquait des êtres de pourriture dissimulés sous les dorures :

She whom the spital-house and ulcerous sores

Would cast the gorge at, this embalms and spices

To th'April day again.

(IV.iii.40-42)

On est tenté, comme le fait John Jowett dans l'édition Oxford d'entendre «spittle» dans ce «spital-house» et de voir là la quintessence d'un monde qui donne la nausée.

Un peu plus tard Apemantus annonce à Timon l'arrivée des bandits en ces termes: "More things like men ; eat Timon, and abhor them » (IV.iii.393). Même si l'on est tenté de penser que le «eat» renvoie Timon à la pauvre racine (« root») qu’il vient de déterrer, les deux injonctions «eat» et «abhor them» semblent être dans le prolongement l'une de l'autre et l'on n'est pas loin d'entendre « eat them and abhor them ». Apemantus lui demande de manger ces hommes, faisant écho à Timon lui-même qui déclarait un peu plus tôt en rongeant sa racine: "That the whole life of Athens were in this! Thus would I eat it» (IV.iii.289-90). Si Timon rêve d'absorber le monde, c'est pour mieux le vomir.

On peut voir, il me semble, dans le deuxième banquet que Timon sert à ses flatteurs un crachat symbolique. Le texte offre bien sûr une littéralisation de l'image du flatteur, lorsqu'en découvrant les mets, Timon lance le spectaculaire: «Uncover, dogs, and lap » (III.vi.72). Mais en servant ce mélange d'eau tiède et d'injures, il crache littéralement au visage de ses invités. "You knot of mouth-friends! Smoke and lukewarm water is your perfection» (III.vi.76-77) ${ }^{39}:$ John Jowett, dans l'édition Oxford, note qu'il y a dans cette évocation un probable écho d'un passage des Révélations (Rev. 3-16): «Because thou art lukewarm, and neither cold nor hot, I will spue thee out of my mouth ». Il semble qu'après s'être repu des flatteurs, après avoir absorbé leur sucre, il les recrache littéralement en leur jetant cette eau tiédasse à la figure («Throwing water in their faces »).

${ }^{39} \mathrm{Au}$ sujet de ce vers, voir Malcolm Ware, "Smoke and Lukewarm Water": A Note in Tim on of Athens », Anglia 82, 1964, p. 342-44. 
La deuxième partie de la pièce, rythmée par les entrées et les sorties des personnages qui rendent visite à Timon, ne fait que prolonger à l'infini ce rejet originel, cette expulsion initiale en forme de crachat. La racine que Timon déterre et dévore est comme l'aliment purgatif qui l'aide à évacuer ce qu'il a sur le cœur, ce qu'il a dans le ventre. C'est à coup de mots qu'il évacue ce qu'il ne peut plus avaler ou digérer. Ces éjections / déjections successives transforment le monde qui l'entoure en «gen'ral excrement» (IV.iii.435). Les trous qu'il creuse dans cette terre souillée sont comme les lieux d'aisance dans lesquels il soulage ses maux / mots de ventre. Si dans la première partie de la pièce le palais de Timon (palace / palate) pouvait absorber un « flot de visiteurs », dans la deuxième partie il les recrache, un à un. Harold Fisch note que le geste de rejet qui consiste à lancer sur les visiteurs des pierres ou de la fange - comme il est parfois suggéré - ce qu'il appelle le « gesture of throwing ${ }^{40}$ » est au cœur de la pièce. Ce geste me semble être l'expression concrète du geste de bouche, de l'acte de langue («speech act ») qui consiste à maudire.

Considérée sous cet angle, la grotte («cave») dans laquelle Timon se réfugie ressemble à une gorge, une bouche ouverte, une cavité, qui ne peut plus rien avaler, qui déverse sur le monde ses déchets. La version BBC mise en scène par Jonathan Miller (1981), montre, lors de la scène des sénateurs, un gros plan sur le visage de Timon. Ce visage que l'on voit à l'envers devient alors illisible pour le spectateur qui ne parvient plus à distinguer que le trou de la bouche au fond d'un autre trou, la grotte. Ce resserrement filmique sur la bouche suggère bien que Timon n'est précisément plus qu'une bouche. Et de cette bouche ouverte, sans barrière, ouverte à tous les vents, émanent les flots d'imprécations si caractéristiques de la pièce.

Une fois seul, Timon déverse en effet l'ordure des mots sur la terre entière. À partir de ce moment, toute l'action de la pièce se trouve dans son mau-dire. La première de ses imprécations commence par l'image d'un mur qui tombe, d'une barrière qui s'effondre :

O thou wall

That girdles in those wolves, dive in the earth

And fence not Athens

${ }^{40}$ Harold Fisch, «Shakespeare and the Language of Gesture », Shakespeare Studies, XIX, 1987, p. 239-51, p. 246. 
Hors du monde et hors de lui, il devient cette langue vagabonde mentionnée par George Webbe dans son traité de 1619 :

the tongue hath long agone been whipt for a Vagabond or a wandring rogue : It goeth (saith the Psalmist) [Psal.59.14,15.] through the earth, it roveth up and down evening and morning, and maketh a noise like a dogge, and wandreth up and down grudging, if it be not satisfied ${ }^{41}$.

George Webbe cite ici le Psaume 59. En devenant ce que les Élisabéthains appellent «a cursetor», Timon devient du même coup «a curser 42 ». Dans son chapitre consacré à « King Lear and The Register of Curse », Kenneth Gross suggère d'ailleurs que le jeu de mots sur «curse» et «cursetor» était sans doute perçu par les contemporains de Shakespeare ${ }^{43}$. «Lingua quo tendis», «Lingua quo vadis?», «Langue, où vas-tu?»: cette question devenue proverbiale ${ }^{44}$, et qu'ont reprise notamment Claude Paradin ${ }^{45}$ et George Wither ${ }^{46}$ un peu plus tard, cette question, on peut se la poser à propos de Timon. Les barrières qui tombent à l'acte IV sont en fait celles de sa bouche, qui n'est plus dorénavant contenue par aucune limite. Il n'est sans doute pas fortuit que le flot d'imprécations qui suit s'ouvre sur l'image d'une incontinence :

[...] Matrons, turn incontinent !

Obedience, fail in children! Slaves and fools,

Pluck the grave wrinkled senate from the bench,

And minister in their steads! To general filths

Convert o'th'instant green virginity!

Do't in your parents' eyes ! Bankrupts, hold fast ;

Rather than render back, out with your knives,

And cut your trusters' throats! Bound servants, steal!

Large-handed robbers your grave masters are,

And pill by law. Maid, to thy master's bed,

\footnotetext{
${ }^{41}$ George Webbe, The Araignement of an Unruly Tongue, Londres, 1619, STC 25156, p. 30. ${ }^{42}$ Voir le deuxième serviteur de Varron, qui donne quasiment une définition du «cursetor » en évoquant la nouvelle situation de Timon : «Who can speak broader than he that has no house to put his head in ? Such may rail against great buildings. » (III.iv.60-61) ${ }^{43}$ Kenneth Gross, Shakespeare's Noise, Chicago et Londres, The University of Chicago Press, 2001 , p. 163.

44 Voir notamment Érasme, La Langue, p. 235 et Adage 39, Collected Works of Erasmus 33, Adage 39, II ii 39 (LB II 460 C-D), p. 93 : «Lingua quo vadis? Tongue, whither wouldst thou? »

45 Claude Paradin, Devises Herö̈ques [1557], intr. Alison Saunders, Aldershot, Scolar Press, 1989, p. 109-110.

${ }^{46}$ George Wither, A Collection of Emblemes [1635], intr. Michael Bath, Aldershot, Scolar Press, 1989, Book 1, illust. XLII, 42.
} 
Thy mistress is o'th 'brothel. Son of sixteen,

Pluck the lined crutch from thy old limping sire,

With it beat out his brains! Piety, and fear,

Religion to the gods, peace, justice, truth,

Domestic awe, night-rest, and neibourhood,

Instruction, manners, mysteries and trades,

Degrees, observances, customs and laws,

Decline to your confounding contraries:

And yet confusion live! Plagues incident to men,

Your potent and infectious fevers heap

On Athens ripe for stroke! Thou cold sciatica,

Cripple our senators, that their limbs may halt

As lamely as their manners! Lust, and liberty,

Creep in the minds and marrows of our youth,

That 'gainst the stream of virtue they may strive,

And drown themselves in riot! Itches, blains,

Sow all th' Athenian bosoms, and their crop

Be general leprosy! Breath infect breath,

That their society, as their friendship, may

Be merely poison! Nothing I'll bear from thee

But nakedness, thou detestable town!

Take thou that too, with multiplying bans!

Timon will to the woods, where he shall find

Th' unkindest beast more kinder than mankind.

The gods confound - hear me, you good gods all -

Th'Athenians both within and out that wall!

And grant, as Timon grows, his hate may grow

To the whole race of mankind, high and low!

Amen.

(IV.i.1-40)

Si je tiens à citer ce texte en entier, aussi indigeste qu'il puisse paraître, c'est pour montrer que dans Timon, comme dans Richard III, «l'infortune» est «prodigue de $\operatorname{mots}^{47}$ », remplie de flots de mots toujours insuffisants qui s'accumulent, coulent d'une ligne à l'autre et débordent des vers. Couper un texte qui, comme les « aræ », poèmes de malédiction antiques repose sur la logique de l'«exemplum», de l'énumération, de l'accumulation, ç'aurait été à la fois en réduire et en proclamer l'excès. Mais il est vrai qu'il s'agit d'un passage dont on est tenté de remplacer la lecture par ces fantômes de texte que sont les « et cætera ». Le texte même invite à cela, puisqu'il formule lui-même la logique du « et cætera»sur laquelle il repose, lorsque Timon déclame : "Take thou that too, with multiplying bans». Par cet appel à des malédictions « sans cesse accrues ${ }^{48} »$, Timon laisse les portes du texte,

\footnotetext{
47 «Why should Calamity be full of words?», King Richard III, IV.iv.126. Traduction de Jean-Michel Déprats, Gallimard, 1995.

48 Traduction de Jean-Michel Déprats, Gallimard, La Pléiade, 2002.
} 
et de sa bouche, ouvertes. Il n'est pas étonnant alors que cette pièce soit souvent considérée comme inachevée: le maudire qui constitue toute l'action des deux derniers actes, le maudire par son fonctionnement verbal même, empêche la clôture. Le "Amen» ironique qui conclut ce texte ne suffit pas à le clore, pas plus d'ailleurs, que les épitaphes de l'acte $v$.

\section{Maudire, mourir, et catera}

«Lips, let four words go by, and language end»(v.ii.210) déclare Timon, quelques vers avant de faire silence. À l'issue de ce parcours, j'aurais tendance bien sûr à préférer la lecture proposée par Rowe : « let sour words go by », au «four words » proposé par la plupart des édition $\mathrm{s}^{49}$. Au-delà de cette lecture, ce vers semble montrer qu'après s'être «soulagé » sur le monde, Timon est maintenant vide de mots, comme s'il avait épuisé toutes ses ressources vitales et verbales, voire tous les mots du monde, et tout cela en vain ${ }^{50}$.

John Jowett, dans l'édition Oxford, signale que dans une mise en scène de $1978^{51}$, le personnage de Timon s'arrachait la langue à la fin de la scène. On peut interpréter cette excrétion physique de la langue, comme le signe d'un dégoût pour le langage et pour ses perversions. Après avoir été dégoûté du monde, Timon est dégoûté de la langue-même. Il est "é-cœuré » par cette langue fétide dont il est devenu, malgré lui, pourtant, l'incarnation.

Timon est en effet devenu cette malebouche qui maudit et pervertit aussi la langue en mélangeant le doux et l'amer, le miel et le fiel. Cette perversion de la langue est mise en scène au cours de trois séquences principales. Au cours du dialogue avec les prostituées d'Alcibiade (IV.iii.1-177) et de l'échange avec les bandits (IV.iii.394449), Timon maudit et bénit d'un même souffle : «Poursuivez », dit-il aux femmes, «Poursuivez votre œuvre de corruption, vous avez ma bénédiction pour continuer d'épandre votre ordure sur le monde». Adressé aux bandits, le mot «honest» est vidé et dévié de son sens

\footnotetext{
49 De même, nous aurions tendance à préférer « obloquy » à « obliquy » (IV.iii. 18).

${ }^{50}$ La pièce insiste à plusieurs reprises sur la vanité des mots, que ce soit des supplications ou des imprécations. Le pire étant déjà sur terre, c'est comme si la question de l'efficacité du mot ne se posait plus.

${ }^{51}$ Oregon Shakespeare Festival, 1978. Timon était joué par Michael Kevin (note de John J owett, éd. Oxford, p. 315)
} 
pour devenir injure, Timon, là encore, louant, bénissant les voleurs tout en les maudissant. Au cours de la scène des sénateurs, Timon fait connaître à ses visiteurs, comme en accéléré, le choc gustatif qu'il a luimême subi au troisième acte. Il couvre de miel le fiel de ses propos en pratiquant la malédiction différée ${ }^{52}$. Aux sénateurs qui viennent lui demander de sauver Athènes d'Alcibiade, il dit «oui» pour mieux pouvoir dire «non », il fait goûter au doux pour pouvoir faire éclater l'amer. Comme le voyageur de la fable d'Ésope, il souffle le chaud pour mieux exhaler le froid.

Dans Timon of Athens la langue est mauvaise et le bon Flavius seul ne peut la racheter. Pour Timon, cette langue n'est plus qu'une douleur. Tel le mauvais riche qui, dans la Bible (Luc 16.24) (53 $^{5}$, suppliait Abraham d'apaiser sa langue, une langue qui avait été si pécheresse qu'elle était condamnée à subir les feux de l'enfer, Timon semble vouloir arrêter le feu qui le consume. L'évocation de la langue pécheresse condamnée au châtiment du feu apparâ̂t lorsque Timon accueille les sénateurs en ces termes :

Thou sun that comforts, burn ! Speak and be hanged ;

For each true word, a blister, and each false

$\mathrm{Be}$ as a cantherising to the root of th'tongue,

Consuming it with speaking.

Ces vers rappellent les châtiments qui étaient infligés aux mauvaises langues, notamment aux blasphémateurs qui, sous Louis IX, par exemple, étaient condamnés à des peines corporelles, et à avoir la langue percée au fer rouge. L'ironie veut que ce soit Timon, devenu l'incarnation de tous les péchés de la langue et dont la bouche est aussi corrompue que le fruit («medlar») qu'Apemantus voudrait lui faire manger (IV.iii.310), qui se fasse ici juge. Timon découvre dans cette pièce que la bonne langue n'existe pas. Aussi n'est-il pas étonnant que, quelques vers plus loin, il condamne à mort le langage (« and language end »). La langue s'est consumée à force d'excès (« consuming it with speaking»). Aussi pourrait-on dire d'elle ce qu'au tout début de la

\footnotetext{
$52 \mathrm{Ou}$ ce qu'un linguiste a appelé «aggravated impoliteness». See Juhani Rudanko, "Aggravated Impoliteness and two types of speaker intention in an episode in Shakespeare's Timon of Athens », Journal of Pragmatics 38, 2006, p. 829-41.

53 Cité par William Perkins, en adresse au lecteur dans A Direction for the Government of the Tongue.
} 
pièce le poète dit du monde. Lorsque le peintre lui demandait : « How goes the world ?», il répondait : «It wears, sir, as it grows » (I.i.3).

Mais au-delà de la mort, l'écrit prend le relais de cette langue qui maudit. Les épitaphes rédigées par Timon, telles les tables de malédictions antiques ou «defixiones», décrites notamment par Lindsay Watson dans son ouvrage Arae. The Curse poetry of Antiquity $(1991)^{54}$, ces épitaphes font appel au pouvoir magique du mot mais ils figent, fixent également une langue devenue débordante. Leur brièveté est comme la manifestation de l'instauration d'une nouvelle économie linguistique. Après avoir coulé à flots, les mots se figent, se fixent dans la cire que le soldat apporte à Alcibiade.

Cependant le texte de Timon d'Athènes nie cette clôture par l'écrit de deux façons au moins. Le mystère textuel que constitue le doublon d'épitaphes interdit toute fixation des mots et du sens. Ce trop plein d'épitaphes rend le texte instable, fuyant, «slippery». D'autre part les mots de la seconde épitaphe font résonner, au-delà de la mort, la voix de la malédiction :

Here lies a wretched corse, of wretched soul bereft;

Seek not my name; a plague consume you, wicked caitiffs left. Here lie I, Timon, who alive all living men did hate;

Pass by and curse thy fill, but pass and stay not here thy gait.

Même gelés dans la cire, ces mots débordent de toutes parts, se contredisent, se répètent. Le texte s'ouvre au moment même où il se ferme. Et ce qui refait basculer le texte dans la logique de l'excès, c'est le «pass by and curse thy fill»: un texte qui maudit son lecteur en l'enjoignant de maudire, qui le condamne à condamner, ne peut jamais en finir de maudire. Ce qui reste à la fin de Timon, c'est certes le silence mais ce silence bruit d'un assourdissant « et cætera», « et cætera ». La langue est morte, vive la langue.

Nathalie VIENNE-GUERRIN

Université de Rouen

\footnotetext{
${ }^{54}$ Leeds, Francis Cairns, 1991.
} 